\title{
A New Algorithm for Distorted Fingerprints Matching Based on Normalized Fuzzy Similarity Measure
}

\author{
Xinjian Chen, Jie Tian, Senior Member, IEEE, and Xin Yang
}

\begin{abstract}
Coping with nonlinear distortions in fingerprint matching is a challenging task. This paper proposes a novel algorithm, normalized fuzzy similarity measure (NFSM), to deal with the nonlinear distortions. The proposed algorithm has two main steps. First, the template and input fingerprints were aligned. In this process, the local topological structure matching was introduced to improve the robustness of global alignment. Second, the method NFSM was introduced to compute the similarity between the template and input fingerprints. The proposed algorithm was evaluated on fingerprints databases of FVC2004. Experimental results confirm that NFSM is a reliable and effective algorithm for fingerprint matching with nonliner distortions. The algorithm gives considerably higher matching scores compared to conventional matching algorithms for the deformed fingerprints.
\end{abstract}

Index Terms-Biometrics, fingerprint, image registration, matching, minutia, similarity measures.

\section{INTRODUCTION}

$\mathbf{S}$ IGNIFICANT improvements in fingerprint recognition have been achieved in terms of algorithms, but there are still many challenging tasks. One of them is matching of nonlinear distorted fingerprints. According to Fingerprint Verification Competition 2004 (FVC2004) [1], they are particularly insisted on: distortion, dry, and wet fingerprints. Distortion of fingerprints seriously affects the accuracy of matching. There are two main reasons contributed to the fingerprint distortion. First, the acquisition of a fingerprint is a three-dimensional/two-dimensional warping process [2], [3]. The fingerprint captured with different contact centers usually results in different warping mode. Second, distortion will be introduced to fingerprint by the nonorthogonal pressure people exert on the sensor. How to cope with these nonlinear distortions in the matching process is a challenging task.

Several fingerprint matching approaches have been proposed in the literature. These include methods based on point pattern matching, transform features and structural matching. Many fingerprint recognition algorithms are based on minutiae matching

Manuscript received Novermber 15, 2004. This paper was supported in part by the Project of National Science Fund for Distinguished Young Scholars of China under Grant 60225008, in part by the Key Project of National Natural Science Foundation of China under Grant 60332010, and in part by the Project for Young Scientists' Fund of National Natural Science Foundation of China under Grant 60303022. The associate editor coordinating the review of this paper and approving it for publication was Dr. Mario A. T. (G. E.) Figueiredo.

The authors are with the Center for Biometrics and Security Research, Key Laboratory of Complex Systems and Intelligence Science, Institute of Automation, Chinese Academy of Sciences, Beijing 100080, China (e-mail: xjchen@fingerpass.net.cn; tian@ doctor.com; yx @ fingerpass.net.cn).

Digital Object Identifier 10.1109/TIP.2005.860597 since it is widely believed that the minutiae are most discriminating and reliable features [4], [5]. Ratha et al. [6] addressed a method based on point pattern matching. The generalized Hough transform (GHT) is used to recover the pose transformation between two impressions. Jain et al. [7], [8] proposed a novel filterbank-based fingerprint feature representation method. Jiang $e t$ al. [9] addressed a method which relies on a similarity measure defined between local structural features, to align the two patterns and calculate a matching score between two minutiae lists. Fan et al. [10] applied a set of geometric masks to record part of the rich information of the ridge structure. Wahab et al. [11] addressed a method using groups of minutiae to define local structural features. The matching is performed based on the pairs of corresponding structural features that are identified between two fingerprint impressions. However, these methods do not solve the problem of nonlinear distortions.

Recently, some algorithms have been presented to deal with the nonlinear distortion in fingerprints explicitly in order to improve the matching performance. Ratha et al. [12] proposed a method to measure the forces and torques on the scanner directly. Which prevents capture with the aid of specialized hardware when excessive force is applied to the scanner. Dorai et al. [13] proposed a method to detect and estimate distortion occurring in fingerprint videos, but those two mentioned methods do not work with the collected fingerprint images. Maio and Maltoni et al. [2] proposed a plastic distortion model to cope with the nonlinear deformations characterizing fingerprint images taken from on-line acquisition sensors. This model helps to understand the distortion process. However, it is hard to automatically and reliably estimate the parameter due to the insufficiency and uncertainty of the information. Dongjae Lee et al. [14] addressed a minutiae-based fingerprints matching algorithm using distance normalization and local alignment to deal with the problem of the nonlinear distortion. However, rich information of the ridge/valley structure is not used, and the matching performance is moderate. Senior et al. [15] proposed a method to convert a distorted fingerprint image into an equally ridge spaced fingerprint before matching to improve the matching accuracy. However, the assumptions of equal ridge spacing is less likely to be true for fingerprints-particularly where ridges break down, such as around minutiae or near the edge of the fingerprint. Watson et al. [16] proposed a method to improve the performance of fingerprint correlation matching using distortion tolerant filters. The performance was improved by using multiple training fingerprints and a distortion-tolerant MINACE filter. However, the algorithm is difficult to realize on 
line. Kov'acs-Vajna et al. [17] also proposed a method based on triangular matching to cope with the strong deformation of fingerprint images, demonstrating graphically that the large cumulative effects can be a result from the small local distortions. Bazen et al. [18] employed a thin-plate spline model to describe the nonlinear distortions between the two sets of possible matching minutiae pairs. By normalizing the input fingerprint with respect to the template, this method is able to perform a very tight minutiae matching.

Different from the above mentioned methods, we propose an algorithm based on fuzzy theory to deal with the nonlinear distortion in fingerprint images. First, an algorithm was defined to deal with the spurious minutiae. Then the algorithm aligns the template and input fingerprints using the registration method described by Luo et al. [19], and local topological structure matching was introduced to improve the robustness of global alignment. Finally a novel similarity computing method based on fuzzy theory, NFSM, was conducted to compute the similarity between the template and input fingerprints. Experimental results indicate that the proposed algorithm works well with the nonlinear distortions. For deformed fingerprints, the algorithm gives considerably higher matching scores compared to conventional matching methods. In fingerprints database DB1 and DB3 of FVC2004, the distortion between some fingerprints from the same finger is large, but our algorithm performs well. The equal error rates (EER) are $4.37 \%$ and $1.64 \%$ on DB1 and DB3, respectively.

This paper is organized as follows. Section II indicates out the fingerprint feature representation and extraction. A method is defined to deal with the spurious minutiae. Section III describes the local topological structure matching. Section IV proposes the novel method, NFSM, which is applied to compute the similarity between the template and input fingerprints. The performance of the proposed algorithm is shown by experiments in Section V. Section VI presents our conclusion and discussion.

\section{FEATURE REPRESENTATION AND EXTRACTION}

For an input fingerprint image, the method described by Hong et al. [20] is used to enhance the image, and the thinned ridge map $T$ is obtained. The thinned ridge map is postprocessed by using Luo's method [21]. Then the minutiae set $M$ is detected by using Hong's method [20]. For each $m_{i}$ belongs to $M$, the associate ridges or valleys in $T$ are traced and some sample points $P$ with constant intervals are recorded. The minutiae set and the sample points on the associate ridges and valleys provide information for both alignment and discrimination. An example of the feature set of a live-scan fingerprint is provided in Fig. 1.

A critical and difficult step in fingerprint matching is to reliably extract minutiae from the input fingerprint images. The performance of a minutiae extraction algorithm highly relies on the quality of the input fingerprint images. In an ideal fingerprint image, ridges and valleys alternate and flow in a local constant direction and minutiae are anomalies of ridges, i.e., ridge endings and ridge bifurcations. In such situations, the ridges can be easily detected and minutiae can be precisely located from the thinned ridges. However, in reality, approximately $10 \%$ [20] of acquired fingerprint images are of poor quality due to variations



(a)

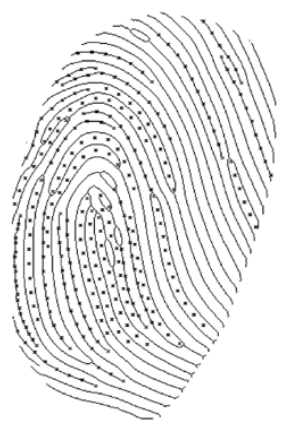

(b)
Fig. 1. Feature set of a live-scan fingerprint image. (a) Original fingerprint image. (b) Thinned ridge image with minutiae and sample points of (a).

in impression conditions, ridge configuration, skin conditions, acquisition devices, and noncooperative attitude of subjects, etc. The ridge structures in poor-quality fingerprint images are not always well-defined and, therefore, cannot be correctly detected. A significant number of spurious minutiae may be created as a result. In order to ensure that the performance of the minutiae extraction algorithm will be robust with respect to the quality of input fingerprint images, an enhancement algorithm which can improve the clarity of the ridge structures is necessary. However, for poor fingerprint image, some spurious minutiae may still exist after fingerprint enhancement and postprocessing. It is necessary to propose a method to deal with the spurious minutiae.

A method to judge whether an extracted minutia is a true one has been proposed in this paper. According to our work, the distance between true minutiae is generally greater than threshold (thre). While near the spurious minutiae, there are usually other spurious minutiae. On the other hand, spurious minutiae are usually detected at the border of fingerprint image. Examples of spurious minutiae in poor quality fingerprint images are shown in Fig. 2.

The following algorithm is performed to detect the spurious minutiae. In this process, a few true minutiae may be considered as spurious ones, but this does not affect further match process.

Step 1: Judge whether the minutia is close to the border of the fingerprint image. The fingerprint mask is obtained by using Hong's algorithm [20]. The distance disMB between the minutiae and the fingerprint border is computed. If disMB $<T 1$ (according to our work, $T 1=5$ ), then it is considered as spurious minutia, else goto step 2 .

Step 2: Search minutiae center around the minutia $M i$ within thre radius, obtain the number of minutiae num.

Step 3: If num $\geq T 2$ (according to our work, $T 2=3$ ), then it is considered as spurious minutia, otherwise as a true one.

It is an efficient and simple method to detect the spurious minutiae. None of the detected spurious minutiae are participated in the further match process. 


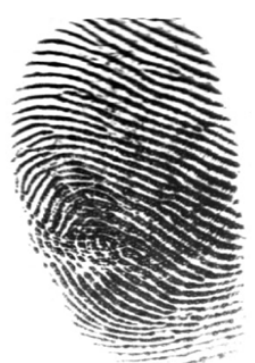

(a)



(c)



(b)



(d)
Fig. 2. Examples of spurious minutiae in poor quality fingerprint images. The images have been cropped and scaled for view. (a) Original image. (b) Enhanced image of (a), (c) original image, (d) enhanced image of (c). Many spurious minutiae were detected in the process of minutiae extraction. Near the spurious minutiae, there are usually other spurious minutiae as indicated in ellipses, and spurious minutiae are usually detected at the border of fingerprint image as shown in rectangles.

\section{MATChing Using LocAl TOPOLOGICAL StruCTURE}

The proposed matching algorithm has two steps. First, Luo's matching method [19] which modified from Jain et al.'s algorithm [22] has been used to process the coarse match. A changeable bounding box is applied during the matching process which makes it more robust to nonlinear deformations between the fingerprint images. Fig. 3 shows the illustrative diagram for changeable sized bounding box. The value of angle_size and radius_size of the bounding box in every minutia is changed according to the radius of the minutia. If the radius of the template minutia is larger, then its bounding box will have a larger radius_size and a smaller angle_size. We suppose an approximate alignment has already been established at this stage. If the approximate alignment is wrong, the further matching steps cannot recover this error. Then, local topological structure match is introduced to improve the robustness of match.

\section{A. Defining of Local Topological Structure}

Let $P$ denote a minutia in the fingerprint image and $Q_{1}, Q_{2}, \ldots, Q_{n}$ are the minutiae circled around $P$ within $r$ radius. Feature vector $\left(\operatorname{len}_{p q_{i}}, \beta_{p q_{i} 1}, \beta_{p q_{i} 2}\right)$ is used to describe the relationship between $P$ and $Q_{i} \cdot \operatorname{len}_{p q_{i}}$ denotes the distance between $P$ and $Q_{i} \cdot \beta_{p q_{i} 1}$ denotes the angle between the orientation of minutia $P\left(\theta_{p}\right)$ and the direction from $P$ to $Q_{i} \cdot \beta_{p q_{i}} 2$ denotes the angle between the orientation of minutia $Q_{i}\left(\theta_{Q_{i}}\right)$ and the direction from $Q_{i}$ to $P$. It is easy to see that the local topological structure feature vector $\left(\operatorname{len}_{p q_{i}}, \beta_{p q_{i} 1}, \beta_{p q_{i} 2}\right)$ is independent from the rotation and translation of the fingerprint.



Fig. 3. Illustrative diagram for changeable sized bounding box.

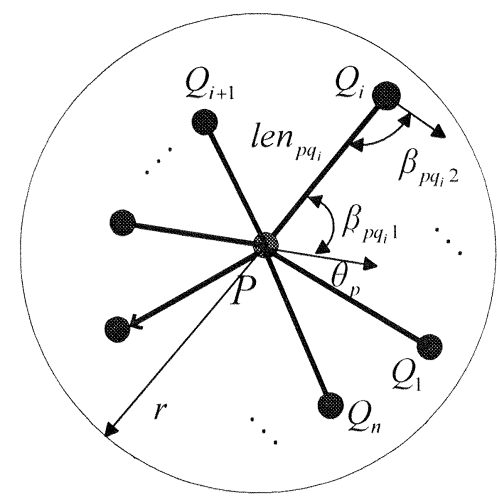

Fig. 4. Local topological structure of $P$.

Fig. 4 shows the meaning of each parameter. The local topological structure of $P$ is defined as the following:

$$
\begin{array}{r}
\operatorname{LoTopo}_{P}=\left(\left(\operatorname{len}_{p q_{1}}, \beta_{p q_{1} 1}, \beta_{p q_{1} 2}\right),\left(\operatorname{len}_{p q_{2}}, \beta_{p q_{2} 1}, \beta_{p q_{2} 2}\right)\right. \\
\left.\ldots,\left(\operatorname{len}_{p q_{n}}, \beta_{p q_{n} 1} \beta_{p q_{n} 2}\right)\right) \\
.
\end{array}
$$

\section{B. Local Topological Structure Matching}

Suppose $P$ is a minutia in the template fingerprint, $Q_{1}, Q_{2}, \ldots, Q_{n}$ are minutiae circled around $P$ within $r$ radius. Then, the local topological structure of $P$ can be obtained as

$$
\begin{array}{r}
\operatorname{LoTopo}_{P}=\left(\left(\operatorname{len}_{p q_{1}}, \beta_{p q_{1} 1}, \beta_{p q_{1} 2}\right),\left(\operatorname{len}_{p q_{2}}, \beta_{p q_{2} 1}, \beta_{p q_{2} 2}\right)\right. \\
\left.\ldots,\left(\operatorname{len}_{p q_{n}}, \beta_{p q_{n} 1}, \beta_{p q_{n} 2}\right)\right) .
\end{array}
$$

Suppose $R$ is a minutia in the input fingerprint and the input fingerprint is deformed. For the deformed input fingerprint, minutiae are searched in a circle around $R$ within $r+r_{s}$ radius. $r_{s}$ is the distance tolerance. Suppose $S_{1}, S_{2}, \ldots, S_{m}$ are minutiae circled around $R$ within $r+r_{s}$ radius. Then, the local topological structure of $R$ can be expressed as

$$
\begin{array}{r}
\operatorname{LoTopo}_{R}=\left(\left(\operatorname{len}_{r s_{1}}, \beta_{r s_{1} 1}, \beta_{r s_{1} 2}\right),\left(\operatorname{len}_{r s_{2}}, \beta_{r s_{2} 1}, \beta_{r s_{2} 2}\right)\right. \\
\left.\ldots,\left(\operatorname{len}_{r s_{m}}, \beta_{r s_{m} 1}, \beta_{r s_{m} 2}\right)\right) .
\end{array}
$$

The following algorithm is used to determine whether two minutiae $P$ and $R$ are matched.

Step 1: For $i(1 \leq i \leq n)$ and $j(1 \leq j \leq m)$, matched each entry $P Q_{i}\left(\operatorname{len}_{p q_{i}}, \beta_{p q_{i} 1}, \beta_{p q_{i} 2}\right)$, in the local topological structure $\operatorname{LoTopo}_{P}$ and each entry $R S_{j}\left(\operatorname{len}_{r s_{j}}, \beta_{r s_{j} 1}, \beta_{r s_{j} 2}\right)$, in 


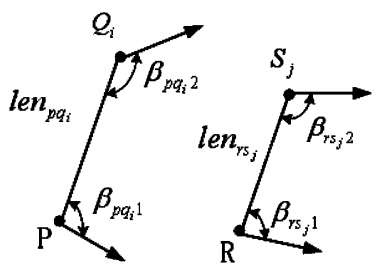

(a)

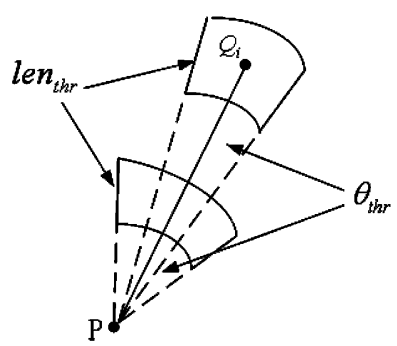

(b)
Fig. 5. Illustrative diagram for matching $P Q_{i}\left(\operatorname{len}_{p q_{i}}, \beta_{p q_{i} 1}, \beta_{p q_{i} 2}\right)$, and $R S_{j}\left(\operatorname{len}_{r s_{j}}, \beta_{r s_{j} 1}, \beta_{r s_{j}}\right)$. (a) Topological structure of $P Q_{i}$ and $R S_{j}$. (b) The adaptive matching bounding box.

the local topological structure LoTopo $_{R}$, obtained the matched number $n_{\text {match } 1}$ of $P Q_{i}$ and $R S_{j}$.

Three parameters $\operatorname{len}_{\text {diff }}, \beta_{1 \text { diff }}, \beta_{2 \text { diff }}$ are calculated as the following:

$$
\begin{aligned}
\operatorname{len}_{\text {diff }} & =\left|\operatorname{len}_{p q_{i}}-\operatorname{len}_{r s_{j}}\right| \\
\beta_{1 \text { diff }} & =\left|\beta_{p q_{i} 1}-\beta_{r s_{j} 1}\right| \\
\beta_{2 \text { diff }} & =\left|\beta_{p q_{i} 2}-\beta_{r s_{j} 2}\right| .
\end{aligned}
$$

An adaptive matching bounding box $M_{b o x}=\left(\operatorname{len}_{\mathrm{thr}}, \beta_{\mathrm{thr}}\right)$ is used to determine whether $P Q_{i}$ and $R S_{j}$ is matched. The size of matching box changes according to the distance $\operatorname{len}_{p q_{i}}$ as seen in (4) and (5), shown at the bottom of the page, where $\operatorname{len}_{L}<\operatorname{len}_{H}, \theta_{L}>\theta_{H}$. The purpose for using a changeable sized bounding box is to deal with nonlinear deformation more robustly. Fig. 5 shows the illustrative diagram for matching $P Q_{i}\left(\operatorname{len}_{p q_{i}}, \beta_{p q_{i} 1}, \beta_{p q_{i} 2}\right)$, and $R S_{j}\left(\operatorname{len}_{r s_{j}}, \beta_{r s_{j} 1}, \beta_{r s_{j} 2}\right)$.

When the distance of $P Q_{i}$ is small, a small deformation will mean a large change of the radial angle while the change of radius remains small. In this case the $\theta_{\mathrm{thr}}$ of the bounding box should be larger and the len $\mathrm{thr}_{\mathrm{th}}$ of the bounding box should be smaller. On the other hand, when the distance of $P Q_{i}$ is large, a small change in radial angle will cause a large change in the position of the minutia. The radius can have larger deformation as the accumulation of deformation from all the regions between $Q_{i}$ and $P$ [17]. In this case, the len $_{\mathrm{thr}}$ of the bounding box should be larger and the $\theta_{\text {thr }}$ should be smaller.

If (1)-(3) satisfy the following conditions:

$$
\begin{aligned}
\operatorname{len}_{\mathrm{diff}} & <\operatorname{len}_{\mathrm{thr}} \\
\beta_{1 \mathrm{diff}} & <\theta_{\mathrm{thr}} \\
\beta_{2 \mathrm{diff}} & <\theta_{\mathrm{thr}}
\end{aligned}
$$

then $P Q_{i}$ and $R S_{j}$ are considered as matched.

Step 2: If $n_{\text {match } 1 / n>T h r_{\text {topo }}}$ then goto Step 3, else $P$ and $R$ are considered as not matched.



(a)

(b)

Fig. 6. Two cases of the local topological structure of $P$ and $R$ satisfy the requirement of step2. (a) Minutia $P$ is actually not matched with minutia $R$. (b) Minutia $P$ is matched with minutia $R$.

Step 3: Fig. 6 shows two cases that the local topological structure of $P$ and $R$ satisfy the requirement of step2. In case (a), there are three minutiae $Q_{1}, Q_{2}, Q_{3}$ circled around $P$ within $r$ radius and $r+2^{*} r s$ radius, but there are six minutiae $S_{1}, S_{2}, \ldots, S_{6}$ circled around $R$ with $r+r s$ radius. Although $n_{\text {match } 1} / n=3 / 3=1$, minutia $P$ is actually not matched with minutia $R$. In case (b), there are three minutiae $Q_{1}, Q_{3}, Q_{5}$ circled around $P$ within $r$ radius but six minutiae $Q_{1}, Q_{2}, \ldots, Q_{6}$ circled around $P$ within $r+2^{*} r s$ radius, and there are six minutiae $S_{1}, S_{2}, \ldots, S_{6}$ circled around $R$ with $r+r s$ radius. As the fingerprint is deformed, minutia $P$ is considered as matched with minutia $R$.

Therefore, we need to handle the above two cases. We obtained another two local topological structure of $P\left(\right.$ LoTopo $\left._{P 2}\right)$ and $R\left(\right.$ LoTopo $\left._{R 2}\right)$. ( LoTopo $\left._{R 2}\right)$ is obtained by searching minutiae circled around $R$ within $r$ radius. Suppose these minutiae are $S_{1}, S_{2}, \ldots, S_{l}$. Then the local topological structure of $R$ LoTopo $_{R 2}$ can be expressed as

$$
\begin{array}{r}
\operatorname{LoTopo}_{R 2}=\left(\left(\operatorname{len}_{r s_{1}}, \beta_{r s_{1} 1}, \beta_{r s_{1} 2}\right),\left(\operatorname{len}_{r s_{2}}, \beta_{r s_{2} 1}, \beta_{r s_{2} 2}\right)\right. \\
\left.\ldots,\left(\operatorname{len}_{r s_{1}}, \beta_{r s_{1} 1}, \beta_{r s_{1} 2}\right)\right) .
\end{array}
$$

LoTopo $_{P 2}$ is obtained by searching minutiae circled around $P$ within $r+r_{s}$ radius. Suppose these minutiae are $Q_{1}, Q_{2}, \ldots, Q_{n}, Q_{n+1}, \ldots, Q_{n^{\prime}}$. Then, the local topological structure of $P \operatorname{LoTopo}_{P 2}$ can be expressed as

$$
\begin{array}{r}
\operatorname{LTopop}_{2}=\left(\left(\operatorname{len}_{p q_{1}}, \beta_{p q_{1} 1}, \beta_{p q_{1} 2}\right),\left(\operatorname{len}_{p q_{2}}, \beta_{p q_{2} 1}, \beta_{p q_{2} 2}\right)\right. \\
\left.\ldots,\left(\operatorname{len}_{p q_{n}}, \beta_{p q_{n} 1}, \beta_{p q_{n} 2}\right)\right) .
\end{array}
$$

Then, using the algorithm described in step 1), match the local topological structure $\operatorname{LoTopo}_{R 2}$ and $\operatorname{LoTopo}_{P 2}$, obtain the matched number $n_{\text {match2 }}$.

Step 4: If $n_{\text {match2 }} / l>T h r_{\text {topo }}$, then $P$ is matched with $R$, else not matched.

Experiments were performed to evaluate the contribution of the proposed local topological structure match algorithm on

$$
\begin{aligned}
\operatorname{len}_{\mathrm{thr}} & = \begin{cases}\operatorname{len}_{L}, & \text { if } \operatorname{len}_{p q_{i}}<T h r_{L} \\
\operatorname{len}_{H}, & \text { if } \operatorname{len}_{p q_{i}}>T h r_{H} \\
\operatorname{len}_{L}+\frac{\mathrm{len}_{H}-\mathrm{len}_{L}}{\mathrm{thr}_{\mathrm{H}}-\mathrm{Thr}_{\mathrm{L}}} \cdot\left(\operatorname{len}_{p q_{i}}-T h r_{L}\right), & \text { otherwise }\end{cases} \\
\theta_{\mathrm{thr}} & = \begin{cases}\theta_{L}, & \text { if len } \\
\theta_{H}, & \text { if } q_{i}<T r_{L} \\
\theta_{L}+\frac{\theta_{H}-\theta_{L}}{\operatorname{thr}_{\mathrm{H}}-\operatorname{Thr}_{\mathrm{L}}} \bullet T h r_{H}\end{cases}
\end{aligned}
$$


TABLE I

COMPARISON OF THE ALGORITHM With AND Without the PROPOSED LOCAL TOPOLOGICAL STRUCTURE MATCH ON FVC2002 DB

\begin{tabular}{|c|c|c|c|c|}
\hline Comparision & $\begin{array}{c}\text { EER (On } \\
\text { FVC2002 } \\
\text { DB1) }\end{array}$ & $\begin{array}{c}\text { EER (On } \\
\text { FVC200 }\end{array}$ & $\begin{array}{c}\text { EER (On } \\
\text { FVC200 }\end{array}$ & $\begin{array}{c}\text { EER(On } \\
\text { FVC200 } \\
\text { 2 DB3) }\end{array}$ \\
\hline $\begin{array}{c}\text { Algorithm without local } \\
\text { topological structure } \\
\text { match }\end{array}$ & $1.03 \%$ & $0.65 \%$ & $1.39 \%$ & $0.88 \%$ \\
\hline $\begin{array}{c}\text { Algorithm with local } \\
\text { topological structure } \\
\text { match }\end{array}$ & $0.19 \%$ & $0.14 \%$ & $0.63 \%$ & $0.15 \%$ \\
\hline
\end{tabular}

FVC2002 DB. Table I illustrates the comparison of the algorithm with and without the proposed local topological structure match on FVC2002 DB. It is clear that the proposed local topological structure match algorithm has obviously improved the overall performance.

\section{NormaLIZED FuZZY Similarity MEASURE}

Computing the similarity between deformed template and input fingerprints is a difficult task. In some algorithms [3], [18], the number of matching minutiae was only used to compute the similarity between template and input fingerprints. Most algorithms increase the size of the bounding boxes in order to tolerate the further apart of matched minutiae pairs because of plastic distortions and, therefore, to decrease the false rejection rate (FRR). However, as a side effect, this method may lead higher false acceptance rate (FAR) by making nonmatching minutiae get paired. Different from the above mentioned algorithm, we proposed a novel similarity computing method based on fuzzy theory to calculate the similarity between template and input fingerprints images.

\section{A. Feature Representation of Similarity Measure}

Two features are selected: the number of matched sample points $(n)$ and the mean distance difference of the matched minutiae pairs $(d)$ in the process of similarity computing. Using the proposed algorithm defined in Section III, the matched minutiae pairs between template and input fingerprints can be obtained. The corresponding sample points are considered to match if their associated minutiae are matched. If the number of sample points for one minutia in a pair is different from the second minutia of the pair, we set the smaller number as the number of matched sample points. Suppose there are $n_{1}$ matched minutiae pairs, and there are $s_{i}$ matched sample points for every minutiae pair, then the sum $n$ of matched sample points is calculated as follows:

$$
n=\sum_{i=1}^{n_{1}} s_{i}
$$

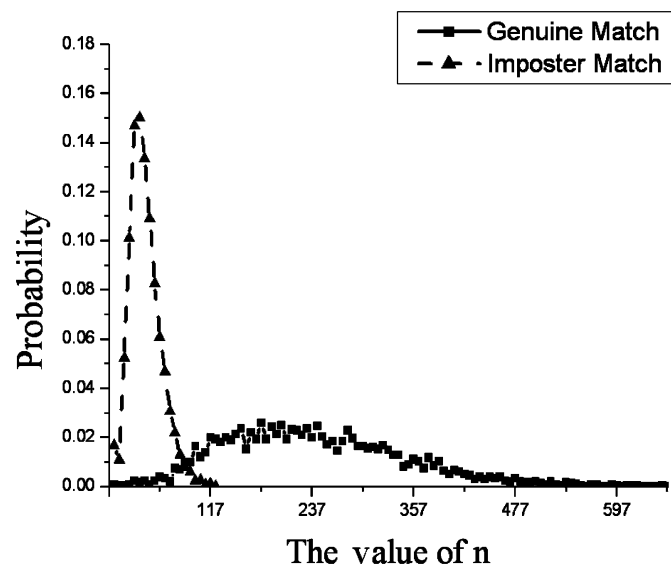

Fig. 7. Probability distribution of $n$ in Genuine match and Imposter match on FVC2002 DB1.

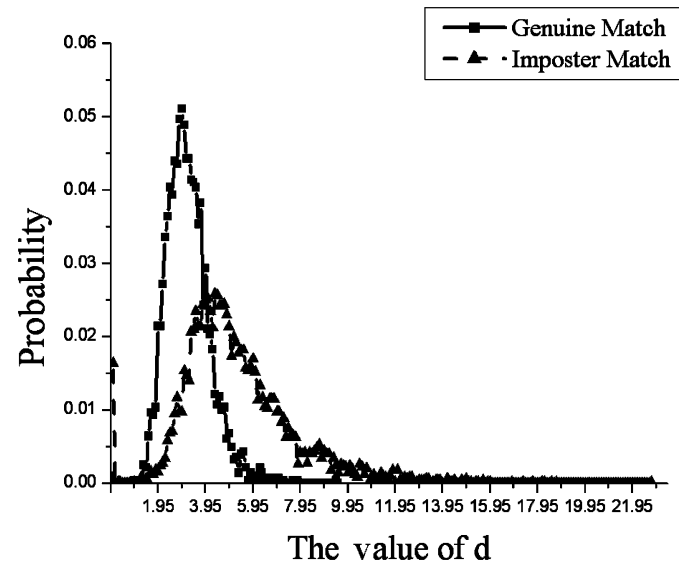

Fig. 8. Probability distribution of $d$ in Genuine match and Imposter match on FVC2002 DB1.

and the mean of distance difference between every two minutiae is calculated as follows:

$$
d=\frac{1}{n_{1} \bullet\left(n_{1}-1\right)} \sum_{i=1}^{n_{1}} \sum_{j=1(j \neq i)}^{n_{1}} \operatorname{len}_{\mathrm{diff}} .
$$

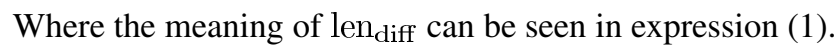

Experiments were performed to characterize the features: $n$ and $d$. The experiments were done on FVC2002 DB1. It contains 800 fingerprint images captured by optical sensor "Identix TouchView II." According to FVC rules [1], each sample is matched against the remaining samples of the same finger in genuine match. Hence, the total number of genuine tests (in case no enrollment rejections occur $)$ is: $((8 * 7) / 2) * 100=2800$. In imposter match, the first sample of each finger is matched against the first sample of the remaining fingers. Hence, the total number of false acceptance tests (in case no enrollment rejections occur) is: $((100 * 99) / 2)=4950$. All of $\mathrm{n}$ and $\mathrm{d}$ samples from genuine match and imposter match were used to construct fuzzy feature sets $\tilde{N}$ and $\tilde{D}$, respectively. Figs. 7 and 8 show the probability distribution of $\mathrm{n}$ and $\mathrm{d}$ in genuine match and imposter match on FVC2002 DB1. From Figs. 7 and 8, we find that $n$ has excellent classification performance and the classification performance of $d$ is also good. Fig. 9 shows a scatter plot of $d$ against $\mathrm{n}$ for all examples in genuine match and imposter match 


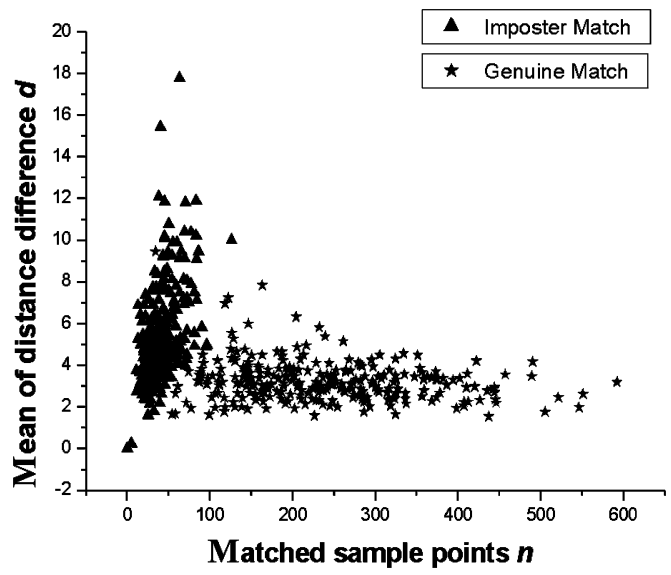

Fig. 9. Scatter plot of $d$ against $n$ for all examples on FVC2002 DB1.

on FVC2002 DB1. The fuzzy feature sets $\tilde{N}$ and $\tilde{D}$ are clustered into two classes. One is corresponding to genuine match samples and the other is to imposter match samples.

\section{B. NFSM Measure}

Fuzzy features were used to represent $n$ and $d$. Each character is associated with a fuzzy feature that assigns a value (between 0 and 1) to each feature vector in the feature space. The value, named degree of membership, illustrates the degree of similarity of the template and input fingerprints.

A fuzzy feature $\tilde{F}$ on the feature space $\Re$ is defined by a mapping $\mu_{\tilde{F}}: \Re \longrightarrow[0,1]$ named as the membership function. For any feature vector $\vec{f} \in \Re$, the value of $\mu_{\tilde{F}}(\vec{f})$ is called the degree of membership of $\vec{f}$ to the fuzzy feature $\tilde{F}$. The value of $\mu_{\tilde{F}}(\vec{f})$ is more close to 1 , the input fingerprint is more similar to the template one. For the fuzzy feature $\tilde{F}$, there is a smooth transition for the degree of membership to $\tilde{F}$ besides the hard cases $\vec{f} \in \tilde{F}\left(\mu_{\tilde{f}}(\vec{f})=1\right)$ and $\vec{f} \notin \tilde{F}\left(\mu_{\tilde{f}}(\vec{f})=0\right)$. It is clear that a fuzzy feature degenerates to a conventional feature set if the range of $\mu_{\tilde{F}}$ is $\{0,1\}$ instead of $[0,1]$.

Building or choosing a proper membership function is an application dependent issue. Some most commonly used prototype membership functions are cone, exponential, and Cauchy functions [23]. In the proposed algorithm, the Cauchy function is chosen due to its good expressiveness and high-computational efficiency [24].

The Cauchy function: $C: \Re^{k} \longrightarrow[0,1]$, is defined as

$$
C(\vec{x})=\frac{1}{1+\left(\frac{\|\vec{x}-\vec{v}\|}{d}\right)^{\alpha}}
$$

where $\vec{v} \in \Re^{k}, d$ and $a \in \Re, d>0, a \geq 0 . \vec{v}$ is the center location of the fuzzy set, $d$ represents the width $(\|\vec{x}-\vec{v}\|)$ for $C(\vec{x})=0.5)$ of the function, and $a$ determines the smoothness of the function. Generally, $d$ and $a$ portray the grade of fuzziness of the corresponding fuzzy feature. For fixed $d$, the grade of fuzziness increases as $a$ decreases. For fixed $a$, the grade of fuzziness increases as $d$ increases.
Accordingly, feature $n$ is represented by fuzzy feature $\tilde{N}$ whose membership function, $\mu_{\tilde{N}}: \Re \longrightarrow[0,1]$, is defined as

$$
\mu_{\tilde{N}}: \Re \longrightarrow[0,1]= \begin{cases}\frac{1}{1+\left(\frac{\left\|\vec{n}-\tilde{N}_{i}\right\|}{d_{n}}\right)^{\alpha 1}}, & n \leq \tilde{N}_{i} \\ 1, & n>\tilde{N}_{i}\end{cases}
$$

where $d_{n}=\left\|\tilde{N}_{g}-\tilde{N}_{i}\right\|$ represents the distance between $\tilde{N}_{g}$ and $\tilde{N}_{i} \cdot \tilde{N}_{g}$ and $\tilde{N}_{i}$ are the genuine and imposter match clusters center of fuzzy set $\tilde{N}_{i}$.

Feature $d$ is represented by fuzzy feature $\tilde{D}$ whose membership function, $\mu_{\tilde{D}}: \Re \longrightarrow[0,1]$, is defined as

$$
\mu_{\tilde{D}}: \Re \longrightarrow[0,1]= \begin{cases}\frac{1}{1+\left(\frac{\left\|d-\tilde{D}_{i}\right\|}{d_{d}}\right)^{\alpha 2}}, & d \leq \tilde{D}_{i} \\ 1, & d>\tilde{D}_{i}\end{cases}
$$

where $d_{d}=\left\|\tilde{D}_{g}-\tilde{D}_{i}\right\|$ represents the distance between $\tilde{D}_{g}$ and $\tilde{D}_{i} \cdot \tilde{D}_{g}$ and $\tilde{D}_{i}$ are the genuine and imposter match clusters center of fuzzy set $\tilde{D}$.

An intrinsic property of membership function (12) and (13) is that the farther a feature vector moves away from the cluster center, the lower the degree of membership is to the fuzzy feature. At the same time, the degrees of membership to the other fuzzy features may be increasing. This clearly describes the gradual transition of two clusters. Table II shows the respective classification performance of $\mu_{\tilde{N}}(\vec{n})$ and $\mu_{\tilde{D}}(\vec{d})$ on FVC2002 DB.

In order to achieve the better classification performance, $\mu_{\tilde{N}}(\vec{n})$ and $\mu_{\tilde{D}}(\vec{d})$ are combined. The detailed analysis on combining classifiers can be seen in the literatures [25]-[27]. After combination, the similarity is needed to normalize to $[0,1]$. Four rules were used to combine $\mu_{\tilde{N}}(\vec{n})$ and $\mu_{\tilde{D}}(\vec{d})$. In these four rules, the similarity is always in the real interval $[0,1]$ because $\mu_{\tilde{N}}(\vec{n})$ and $\mu_{\tilde{D}}(\vec{d})$ are always within $[0,1]$.

1) Max rule:

$$
\text { similarity }=\max \left(\mu_{\tilde{N}}(\vec{n}), \mu_{\tilde{D}}(\vec{d})\right) .
$$

2) Min rule:

$$
\text { similarity }=\min \left(\mu_{\tilde{N}}(\vec{n}), \mu_{\tilde{D}}(\vec{d})\right) .
$$

3) Product rule:

$$
\text { similarity }=\left(\mu_{\tilde{N}}(\vec{n}) * \mu_{\tilde{D}}(\vec{d})\right) .
$$

4) Mean rule:

$$
\text { similarity }=\frac{\left(\mu_{\tilde{N}}(\vec{n})+\mu_{\tilde{D}}(\vec{d})\right)}{2} .
$$

Experiments were performed to evaluate four similarity computing rules on FVC2002 DB. Table II shows the comparison of performance on FVC2002 DB in different similarity computing rules. On DB1, if only $\mu_{\tilde{N}}(\vec{n})$ is used to classify in fingerprint matching, EER is $3.03 \%$, and if only $\mu_{\tilde{D}}(\vec{d})$ is used to classify in fingerprint matching, EER is $20.13 \%$. While using the product rule to compute the similarity, EER is decreased to $0.19 \%$. From Table II, we can clearly tell that the performance 
TABLE II

COMPARISON OF PERFORMANCE ON FVC2002 DB IN DIFFERENT SimILARITY COMPUTING RULES

\begin{tabular}{|c|c|c|c|c|}
\hline $\begin{array}{l}\text { Similarity computing } \\
\text { rules }\end{array}$ & $\begin{array}{l}\text { EER (On } \\
\text { FVC200 } \\
2 \text { DB1) }\end{array}$ & $\begin{array}{l}\text { EER (On } \\
\text { FVC200 } \\
2 \text { DB2) }\end{array}$ & $\begin{array}{l}\text { EER (On } \\
\text { FVC200 } \\
2 \text { DB3) }\end{array}$ & $\begin{array}{l}\text { EER (On } \\
\text { FVC200 } \\
2 \text { DB4) }\end{array}$ \\
\hline$\mu_{N}(n)$ & $3.03 \%$ & $2.02 \%$ & $4.03 \%$ & $1.06 \%$ \\
\hline$\mu_{\widetilde{D}}(d)$ & $20.13 \%$ & $23.16 \%$ & $26.51 \%$ & $17.01 \%$ \\
\hline$\mu_{\tilde{N}}(n) * \mu_{\tilde{D}}(d)$ & $0.19 \%$ & $0.14 \%$ & $0.63 \%$ & $0.15 \%$ \\
\hline $\begin{array}{l}\left(\mu_{\tilde{N}}(n)+\right. \\
\left.\mu_{\tilde{D}}(d)\right) / 2\end{array}$ & $0.38 \%$ & $0.21 \%$ & $0.63 \%$ & $0.17 \%$ \\
\hline $\begin{array}{c}\max \left(\mu_{\tilde{N}}(n)\right. \\
\left.\mu_{\widetilde{D}}(d)\right)\end{array}$ & $3.42 \%$ & $7.42 \%$ & $14.80 \%$ & $13.40 \%$ \\
\hline $\begin{array}{l}\min \left(\mu_{\tilde{N}}(n)\right. \\
\left.\mu_{\widetilde{D}}(d)\right)\end{array}$ & $0.24 \%$ & $0.15 \%$ & $0.63 \%$ & $0.15 \%$ \\
\hline
\end{tabular}
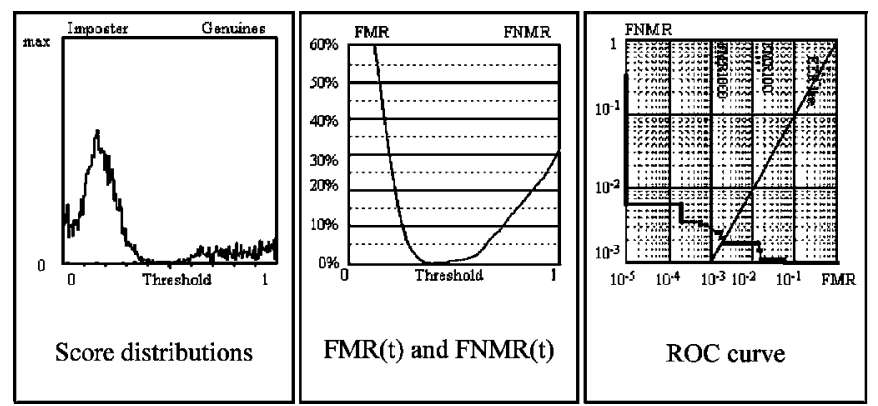

Fig. 10. Experimental results of the proposed algorithm on FVC2002 DB1.

of product rule is the best while the performance of max rule is the worst. Hence, the product rule was selected in the proposed algorithm to compute the similarity between the template and input fingerprints. Fig. 10 shows the experimental results of the proposed algorithm on FVC2002 DB1.

\section{EXPERIMENTAL RESULTS}

The proposed algorithm has been participated in FVC2004. In FVC2004, databases are more difficult than in FVC2000/FVC2002 ones. In FVC2004, the organizers have particularly insisted on: distortion, dry, and wet fingerprints. Especially in fingerprints database DB1 and DB3 of FVC2004, the distortion between some fingerprints from the same finger is

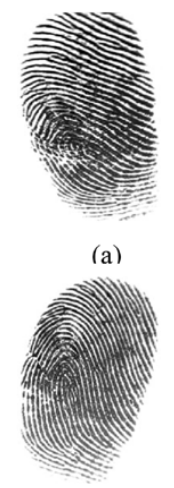

(b)

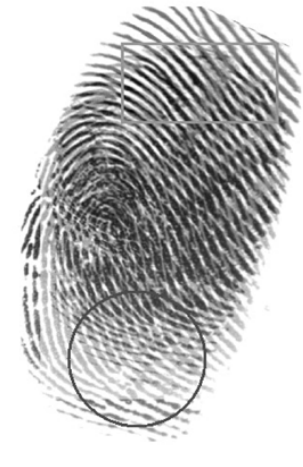

(c)
Fig. 11. Example of large distortion from FVC2004 DB1. (a) 102_3.tif; (b) 102_5.tif; (c) image which (a) (after registration) was added to (b). In the rectangle region, the corresponding minutiae are approximately overlapped. While in the ellipse region, the maximal vertical difference of corresponding minutiae is above 100 pixels.

large. Our work is to solve the problem of distorted fingerprint matching, so the evaluation of the proposed algorithm is mainly focused on DB1 and DB3 of FVC2004. The proposed algorithm is also compared with the one described by Luo et al. [19] and the one proposed by Bazen et al. [18].

\section{A. Performance On FVC2004 DB1}

The fingerprints of FVC2004 DB1 were acquired through CrossMatch V300 (Optical sensor). The size of the image is $640 * 480$ pixels with the resolution about 500 dpi. The fingerprint database set A contains 800 fingerprint images captured from 100 different fingers, eight images for each finger. Fig. 11 shows an example of large distortion from FVC2004 DB1 (102_3.tif and 102_5.tif). While the corresponding minutiae in green region are approximately overlapped, the maximal vertical difference of corresponding minutiae in red region is above 100 pixels. Fig. 12 indicates our enhanced and matched results on 102_3.tif and 102_5.tif. Using the proposed algorithm, the similarity between these two fingerprints is 0.420082 . Fig. 13 presents another fingerprint pair with large distortion from FVC2004 DB1. The similarity between these two fingerprints is 0.650000 . The performance of the proposed algorithm on FVC2004 DB1 is shown in Fig. 14, and from which we find that the similarity threshold at EER point is about 0.28 , so we judge that the above two fingerprint pairs come from the same finger. The two matches are successful. The EER of the proposed algorithm on FVC2004 DB1 is about 4.37\%. The average time for matching two minutiae sets is about 0.77 s on PC AMD Athlon $1600+(1.41 \mathrm{GHz})$.

\section{B. Performance On FVC2004 DB3}

The fingerprints of FVC2004 DB3 were acquired through thermal sweeping sensor "FingerChip FCD4B14CB" by Atmel. The size of the image is $300 * 480$ pixels with the resolution of 512 dpi. In this fingerprints database, the distortion between some fingerprints from the same finger is large. Fig. 15 shows a fingerprint pair with large distortion from FVC2004 DB3. Using the proposed algorithm, the similarity between these two fingerprints is 0.484111 . The performance of the proposed algorithm 


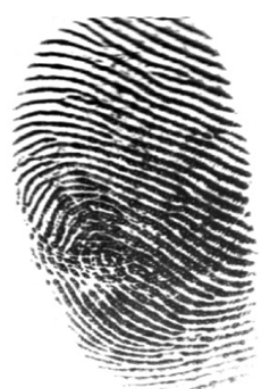

(a)



(c)

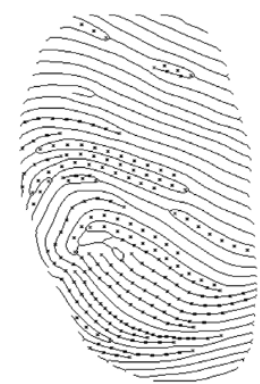

(b)

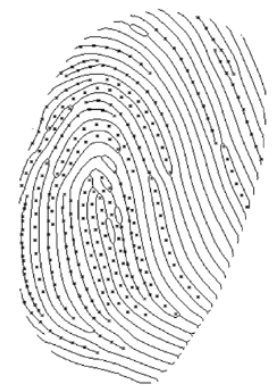

(d)
Fig. 12. Experimental results of the proposed algorithm on 102_3.tif and 102_5.tif in FVC2004 DB1. The images have been cropped and scaled for view. (a) 102_3.tif. (b) Enhanced image of 102_3. (c)102_5.tif. (d) Enhanced image of 102_5. The similarity of these two fingerprints is 0.420082 .

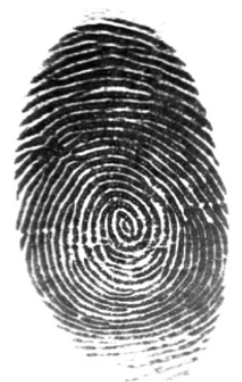

(a)



(c)

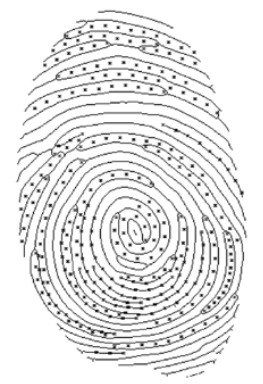

(b)

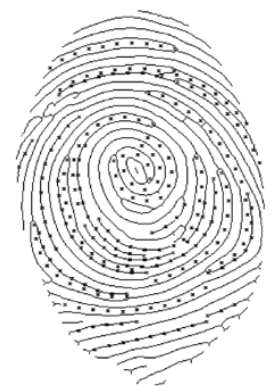

(d)
Fig. 13. Experimental results of the proposed algorithm on 109 3.tif and 109_4.tif in FVC2004 DB1. The images have been cropped and scaled for view. (a) 109_3.tif. (b) Enhanced image of 109_3. (c) 109_4.tif. (d) Enhanced image of 109_4. The similarity of these two fingerprints is 0.650000 .

on FVC2004 DB3 is shown in Fig. 16. It indicates that the similarity threshold at EER point is about 0.26 , so we can judge that the fingerprint pair comes from the same finger. The EER of our algorithm on FVC2004 DB3 is about $1.64 \%$. The average time for matching two minutiae sets is about $0.81 \mathrm{~s}$ on PC AMD Athlon $1600+(1.41 \mathrm{GHz})$.
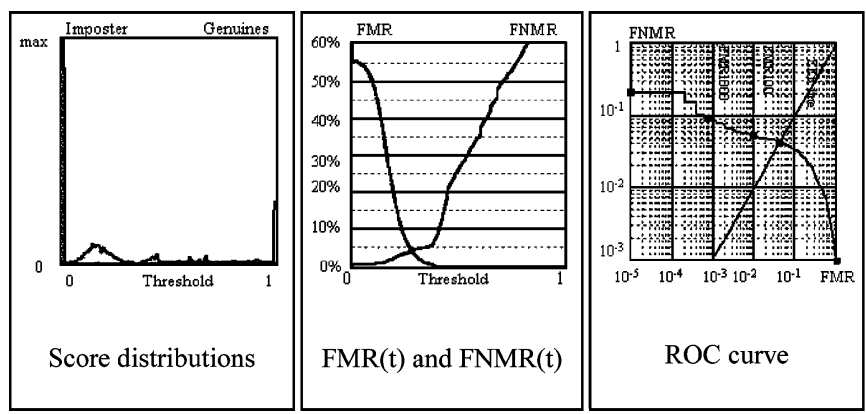

Fig. 14. Experimental results of the proposed algorithm on FVC2004 DB1_A. The fingerprint images were acquired through optical sensor.

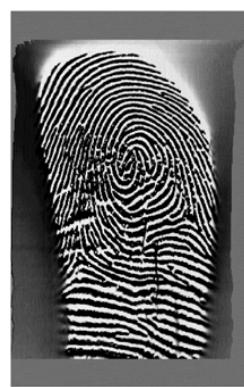

(a)

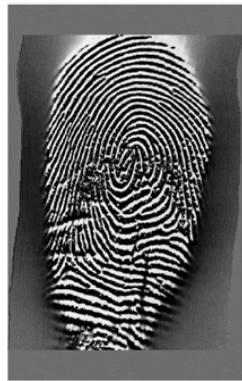

(c)

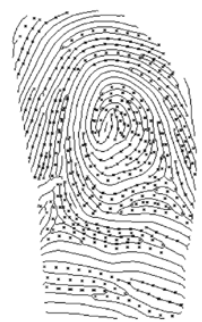

(b)



(d)
Fig. 15. Experimental results of the proposed algorithm on 103_2.tif and 103_4.tif in FVC2004 DB3. The images have been scaled for view. (a) 103_2.tif. (b) Enhanced image of 103_2. (c) 103_4.tif. (d) Enhanced image of 103_4. The similarity of these two fingerprints is 0.484111 .



Fig. 16. Experimental results of the proposed algorithm on FVC2004 DB3_A. The fingerprint images were acquired through thermal sweeping sensor.

The proposed algorithm has good performance on fingerprints database DB2 and DB4 of FVC2004 as well. EER are $2.59 \%$ and $0.61 \%$, respectively. The processing time is 0.58 and $0.56 \mathrm{~s}$, respectively. 
TABLE III

COMPARISON OF MATCHING SCORE IN THE NFSM ALGORITHM TO LUO'S METHOD

\begin{tabular}{|l|l|l|l|}
\hline & The & X. P. Luo's & X. P. Luo's \\
Performance comparison & proposed & of the & method: size \\
& algorithm & bounding & bounding \\
& & boxes $=15$ & boxes $=25$ \\
\hline Score of case 1 in fig. 9 & 0.42008 & 0.05000 & 0.19400 \\
\hline Score of case 2 in fig. 10 & 0.65000 & 0.06180 & 0.27360 \\
\hline Score threshold at EER point & 0.27620 & 0.15200 & 0.21310 \\
\hline EER(on FVC2004 DB1_A) & $4.37 \%$ & $9.13 \%$ & $9.92 \%$ \\
\hline
\end{tabular}



(a)

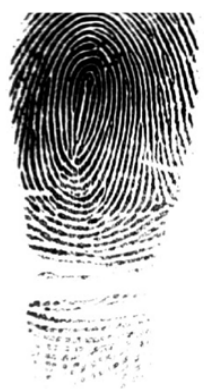

(c)

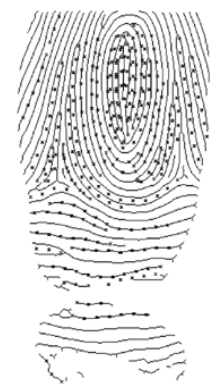

(b)



(d)
Fig. 17. Example of false acceptance on a pair of fingerprints captured from CrossMatch sensor. The images have been cropped and scaled for view. (a) Original image. (b) Enhanced image of (a). (c) Original image. (d) Enhanced image of (c). The fingerprint pair is very similar, but they are from different finger. The similarity of the two fingerprints is 0.431516 .

\section{Comparison of NFSM With Other Algorithm}

The proposed algorithm is compared with the one described by Luo et al. [19]. The Comparison is performed on FVC2004 DB1. In Luo's method, a changeable bounding box was used during the matching process. It is more robust to nonlinear deformations between the fingerprint images. However, the distortion among some fingerprints from the same finger captured from the CrossMatch sensor is too large. In order to tolerate matching minutiae pairs that are further apart because of distortions, the size of the bounding boxes has to be increased. However, as a side effect, it gives a higher probability for those nonmatching minutiae pairs to get paired, resulting in a higher false acceptance rate. Table III lists the comparison of matching score of the proposed algorithm to Luo's method. It is clear that case 1 and case 2 have been successfully matched in our NFSM algorithm. While for Luo's method, when size of the bounding boxes is 15 , case 1 and case 2 are false rejected, and when size of the bounding boxes is increased to 25 , case 2 is accepted but case 1 is false rejected, meanwhile EER is increased from $9.13 \%$ to $9.92 \%$. Moreover, EER of the proposed algorithm is $4.37 \%$, which is much lower than Luo's method of 9.13\%.

The proposed algorithm is also compared with Bazen's method [18]. In their training database of FVC2002 DB1, for Bazen's algorithm, the EER of the ROC turned out to be $1.8 \%$ with $r 0=5$ for elastic matching. While in our algorithm, the EER on FVC2002 DB1 is only about $0.19 \%$. The experimental results of our algorithm on FVC2002 DB1 are shown in Fig. 10. The results conform that the performance of the proposed algorithm surpasses Bazen's algorithm.

\section{CONCLUSION AND Discussion}

Coping with nonlinear distortions in the matching algorithm is a challenging task. In this paper, we proposed a novel algorithm to deal with the nonlinear distortion in fingerprint images. A novel similarity computing method based on fuzzy theory, NFSM, is introduced to compute the similarity between the template and input fingerprints. Experimental results show that the algorithm gives considerably higher matching scores compared to conventional matching algorithms for deformed fingerprints. Our algorithm has been participated in FVC2004. The Participant ID is P071 (open). The detailed performance of the proposed algorithm on FVC2004 can be seen from the website http://bias.csr.unibo.it/fvc2004/default.asp. In fingerprints database DB1 and DB3 of FVC2004, the distortion between some fingerprints from the same finger is large. However, EER are only $4.37 \%$ and $1.64 \%$ on DB1 and DB3, respectively, in our algorithm, and it performs well on processing time, the average time for matching two minutiae sets is about $0.67 \mathrm{~s}$.

However, there are still few false acceptances in fingerprint matching process. Fig. 17 shows an example of false acceptance on a pair of fingerprints captured from CrossMatch sensor. The pair is very similar, but they are from different finger. Further investigation is going on to advance the algorithm for its false acceptance which occasionally happens.

\section{REFERENCES}

[1] Biometric Systems Lab., Pattern Recognition and Image Processing Lab. Biometric Test Center. [Online]. Available: http://bias.csr.unibo.it/fvc2004/

[2] R. Cappelli, D. Maio, and D. Maltoni, "Modeling plastic distortion in fingerprint images," in Proc. ICAPR, Mar. 2001, pp. 369-376.

[3] H. Chen, J. Tian, and X. Yan, "Fingerprint matching with registration pattern inspection," in Proc. AVBPA, 2003, pp. 327-334.

[4] The Science of Fingerprints: Classification and Uses. Washington, DC: Federal Bureau of Investigation, 1984.

[5] H. C. Lee and R. E. Gaensslen, Eds., Advances in Fingerprint Tech nology. New York: Elsevier, 1991.

[6] N. K. Ratha, K. Karu, S. Chen, and A. K. Jain, "A real-time matching system for large fingerprint databases," IEEE Trans. Pattern Anal. Mach. Intell., vol. 18, no. 8, pp. 799-813, Aug. 1996.

[7] A. K. Jain, S. Prabhakar, L. Hong, and S. Pankanti, "Filterbank-based fingerprint matching," IEEE Trans. Image Process., vol. 9, no. 5, pp. 846-859, May 2000.

[8] A. Ross, A. K. Jain, and J. Reisman, "A hybrid fingerprint matcher," Pattern Recognit., vol. 36, no. 7, pp. 1661-1673, 2003. 
[9] X. Jiang and W. Y. Yau, "Fingerprint minutiae matching based on the local and global structures," in Proc. 15th ICPR, vol. 2, 2000, pp. $1038-1041$.

[10] K. C. Fan, C. W. Liu, and Y. K. Wang, "A randomized approach with geometric constraints to fingerprint verification," Pattern Recognit., vol. 33, no. 11, pp. 1793-1803, 2000.

[11] A. Wahab, S. H. Chin, and E. C. Tan, "Novel approach to automated fingerprint recognition," Proc. Int. Elect. Eng. Vis. Image Signal Process., vol. 145 , no. 3, pp. 160-166, 1998.

[12] N. K. Ratha and R. M. Bolle, "Effect of controlled acquisition on fingerprint matching," Proc. 14th ICPR, vol. 2, pp. 1659-1661, 1998.

[13] C. Dorai, N. Ratha, and R. Bolle, "Detecting dynamic behavior in compressed fingerprint videos: Distortion," in Proc. CVPR, Hilton Head, SC, Jun. 2000, pp. 2320-2326.

[14] D. Lee, K. Choi, and J. Kim, "A robust fingerprint matching algorithm using local alignment," in Proc. 16th ICPR, vol. 3, 2002, pp. 803-806.

[15] A. Senior and R. Bolle, "Improved fingerprint matching by distortion removal," IEICE Trans. Inf. Syst., vol. E84-D, no. 7, pp. 825-831, Jul. 2001.

[16] C. Watson, P. Grother, and D. Cassasent, "Distortion-tolerant filter for elastic-distorted fingerprint matching," Proc. SPIE Opt. Pattern Recognit., pp. 166-174, 2000.

[17] Z. M. Kovács-Vajna, "A fingerprint verification system based on triangular matching and dynamic time warping," IEEE Trans. Pattern Anal. Mach. Intell., vol. 22, no. 11, pp. 1266-1276, Nov. 2000.

[18] A. M. Bazen and S. H. Gerez, "Fingerprint matching by thin-plate spline modeling of elastic deformations," Pattern Recognit., vol. 36, no. 8, pp. 1859-1867, 2003.

[19] X. P. Luo, J. Tian, and Y. Wu, "A minutia matching algorithm in fingerprint verification," in Proc. 15th ICPR, vol. 4, 2000, pp. 833-836.

[20] L. Hong, Y. Wan, and A. K. Jain, "Fingerprint image enhancement: Algorithms and performance evaluation," IEEE Trans. Pattern Anal. Mach. Intell., vol. 20, no. 8, pp. 777-789, Aug. 1998.

[21] X. P. Luo and J. Tian, "Knowledge based fingerprint image enhancement," in Proc. 15th ICPR, vol. 4, 2000, pp. 783-786.

[22] A. K. Jain, L. Hong, and R. Bolle, "On-Line fingerprint verification," IEEE Trans. Pattern Anal. Mach. Intell., vol. 19, no. 4, pp. 302-313, Apr. 1997.

[23] F. Hoppner, F. Klawonn, R. Kruse, and T. Runkler, Fuzzy Cluster Analysis: Methods for Classification, Data Analysis and Image Recognition. New York: Wiley, 1999.

[24] Y. X. Chen and J. Z. Wang, "A region-based fuzzy feature match approach to content-based image retrieval," IEEE Trans. Pattern Anal. Mach. Intell., vol. 24, no. 9, pp. 1252-1267, Sep. 2002.

[25] J. Kittler, M. Hatef, P. W. Duin, and J. Matas, "On combining classifiers," IEEE Trans. Pattern Anal. Mach. Intell., vol. 20, no. 3, pp. 226-239, Mar. 1998.

[26] S. Hashem and B. Schmeiser, "Improving model accuracy using optimal linear combinations of trained neural networks," IEEE Trans. Neural Netw., vol. 6, no. 3, pp. 792-794, Jun. 1995.
[27] T. K. Ho, J. J. Hull, and S. N. Srihari, "Decision combination in multiple classifier systems," IEEE Trans. Pattern Anal. Mach. Intell., vol. 16, no. 1, pp. 66-75, Jan. 1994.



Xinjian Chen received the B.S. degree in geophysics from Centre South University of Technology, China, in 2001. He is currently pursuing the Ph.D. degree at the Institute of Automation, Chinese Academy of Sciences, Beijing.

His research interests include pattern recognition, machine learning, image processing, and their applications in biometrics.

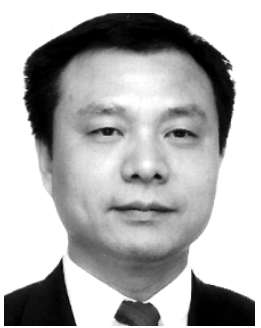

Jie Tian (SM'02) received the Ph.D. degree (with honors) in artificial intelligence from the Institute of Automation, Chinese Academy of Sciences, Beijing, in 1992.

From 1994 to 1996, he was a Postdoctoral Fellow with the medical image processing group, University of Pennsylvania, Philadelphia. Since 1997, he has been a Professor with the Institute of Automation, Chinese Academy of Sciences. His research interests include pattern recognition, machine learning, image processing and their applications in biometrics, etc.



Xin Yang received the Ph.D. degree in precision instruments from TianJing University, China, in 2000.

She is a Postdoctoral Fellow with Institute of Automation, Chinese Academy of Sciences, Beijing. Her research interests include pattern recognition and its applications in biometrics. 